techniques, perhaps best illustrated by the fact that the existence of galaxies was recognized by optical observations, but would now independently be found from radio observations. Putting $A=35, B=7$ in 1979 , with one triply recognized phenomenon $(C=1)$, he estimates that there are 123 separate cosmic phenomena to be found of which we have now recognized 35 per cent. From a discussion of the rate of discovery he thinks we might know all of them by $2150 \mathrm{AD}$, a somewhat depressing conclusion for those who love observational astronomy, though favourable error bars would increase the numbers and postpone the date. Harwit distinguishes between original discoveries and their development, so that our successors may not all be unemployed by then.

$\mathrm{He}$ builds upon a figure published by Halton Arp in 1965 which defines the limitations of observations of extended visual sources considered in terms of their absolute magnitudes and linear diameters. In an elaborate analysis of techniques he considers the parameters of possible detection methods - frequency, size, spectral resolution, time resolution, ellipticity of polarization and intensity - and constructs multidimensional phase diagrams to show what is now accessible and what might become accessible in the future, and classifies each of his fundamental discoveries according to this scheme.

One chapter outlines his selected set of phenomena and the circumstances of their discovery. He makes a number of points in passing - for example that new discoveries follow the introduction of new techniques, with which few readers will quarrel. However he tends to think only of revolutions in techniques rather than in their availability. To an observer one of the most important events of the past few decades was the identification by Thackeray and Wesselink of both Cepheids and RR Lyrae stars in the Magellanic Clouds because of the newly available 74-inch reflector in the Southern Hemisphere. The resultant doubling of the scale of the Universe does not rank in his list of 43 discoveries. He mentions the serendipity of many discoveries, but fails to mention the outstanding discovery by John B. Irwin that S Normae was a member of the cluster NGC 6087 . In fact his accounts of pulsating variables and flare stars leave a good deal to be desired and he fails to mention that pulsations continue down even into the white dwarf range. Indeed, one has the impression that in his accounts of fundamental discoveries Harwit confines himself to data from a rather restricted selection of well-known sources, especially those closest to the NASA publicity machine. For example, in his remarks on pulsars we fail to find any mention of the names of Cocke, Disney, Taylor, Nather or Warner, and the name of Lundmark is omitted from the story of the identification of the $1054 \mathrm{AD}$ supernova remnant. The independent observation of the Uranus rings by Joseph Churms gets no mention. Among other distinguished absentees, de Vaucouleurs rates no inclusion for the discovery of the local supergalaxy.

Though one can quarrel with Harwit's selection of phenomena and his historical accounts, perhaps this will not invalidate his main thesis for some readers. He remarks that many of the discoverers were not originally trained as astronomers, which is true but easily explained. The basic discoveries in radio, X-ray or gamma-ray astronomy were made before anybody could describe himself as an astronomer specializing in these fields. Until a few decades ago, employment opportunities in astronomy were so few that even aspirant astronomers took care to have an employable skill in some other field as a hedge against failure in the discipline of first choice. He remarks that by his reckoning the major proportion of recent discoveries has been made by Americans, though he might have added that even now a high proportion of top posts in American astronomy are held by individuals whose original training was received elsewhere, apparently himself included.

Lastly, he notes that many of the most original discoveries have been made by individuals outside the main stream of astronomical organization and funding. This, of course, has been remarked in other sciences; one can command organized research but it seems impossible to command true originality. However, insofar as observational astronomers in all fields are now married to large and expensive instruments, detailed planning and allocation of resources is essential.

The last part of the book contains Harwit's message - "How should we organize astronomy?". He argues for 13 recommendations, many of which are obvious enough - better training in physics and mid-career training in new techniques for astronomers, and vigorous introduction of exponents of new techniques in astronomy. Long-term grants will better allow astronomers to address fundamental problems and the current peer review system must be loosened up to encourage true innovation, "which can seldom be justified in advance". On the other hand, projects should not be kept on just because they have gone on for a long time. He wishes to encourage gravitational wave and neutrino astronomy and believes that in the electromagnetic domain current observing capabilities could be increased by a factor of 1,000 at modest cost. $\mathrm{He}$ finishes with a plea for policy to be set by panels of generalists of "unusual breadth of interest and far-ranging vision", more frequent policy reviews and frequent analyses of the factors which contribute to astronomical success.

These are fine words, but how to implement them in the real world with limited funds for pure research is another matter. In view of Harwit's desire to have more frequent review committees, it is odd that he admits that "there is no evidence . . . that astronomical planning committees have in any way advanced the rate at which new astronomical phenomena are discovered ...". This is the paradox: astronomers need to spend a lot of money, much from public sources, and need to be accountable. They can guarantee the addition of large amounts of extremely valuable knowledge from their efforts. But the history of science demonstrates two things. One is that those who have predicted the exhaustion of the results of research have usually been proven wrong, however logical their arguments. The other is that discoveries of real originality are most often made by people who ignore the basic plan and pursue their own hunches. Many of them are rightly written off as cranks, but those who succeed are geniuses.

David S. Evans is a former Associate Director for Research at the McDonald Observatory and currently a Professor of Astronomy at the University of Texas at Austin.

\section{Relativity for all}

\section{D.J. Raine}

Discovering Relativity for Yourself. By Sam Lilley. Pp.425. ISBN hbk 0-521-23038-1; ISBN pbk 0-521-29780-X. (Cambridge University Press: 1981.) Hbk $£ 17.50, \$ 49.50$; pbk £7.95, \$19.95.

ONE approach to physical science for the intelligent but innumerate layman is through analogy and example, omitting the why and wherefore. Sam Lilley has a different approach. He believes that students can be taught how to work things out for themselves.

Schopenhauer opined that mathematical truths properly presented can, in any case, be grasped intuitively, thereby circumventing the aridity of the Euclidean mode, for which he gave as sole justification a pictogram proof of Pythagoras's theorem. Lilley has a beautiful extension of this to the invariance of the interval in special relativity. But, even when only elementary mathematics is involved, not everything can be presented so easily; for example, on p.74 we must digress from space-time diagrams to explain negative numbers.

With general relativity the difficulties multiply. The equivalence principle is

The latest edition of The Milky Way by Bart and Priscilla Bok has just been published by Harvard University Press. The book, which first appeared in 1941 and has now been revised for the fifth time, costs $£ 14$. 
discussed well in terms of accelerated observers in special relativity. But to master Lilley's "near equations" seems to require as much effort as would elementary calculus proper. Some limitations of the approach are apparent from the author's speculations on an alternative theory. This is incompatible with the precession of Mercury, but the reader (or author) is not provided with the confidence to do the relevant short calculation. Nor does one get any feeling for the results of the theory in cosmology, black holes or gravitational waves.

There are some mistakes: for example, a too-simple proof that bodies cannot move with the speed of light, and a peculiar notion of covariant tensors, which arises from confusing two meanings of "covariant". And amongst the more serious errors, two incompatible equations for the same gravitational force arise from an inconsistent approximation.

But for me the achievement outweighs these flaws of execution. Of the greatest importance is the demonstration, through a large number of well-made teaching points, that mathematics, the language of science, is available to anyone with sufficient motivation and the right guide.

D.J. Raine is a Lecturer in Theoretical Astrophysics at the University of Leicester. His books include Einstein and Relativity (Priory Press, 1975) and The Isotropic Universe (Adam Hilger, 1981).

\section{Can the U.S. stop advancing when Russia is advancing nuclear power?

$$
\text { read: }
$$ \\ FROM SCIENTIFIC SEARCH TO ATOMIC INDUSTRY}

\author{
By A. M. Petrosyants
}

388 pages $6 \times 9$ Hardbound $\$ 17.90$

This is a historical reference book to make the USSR's nuclear science and nuclear power development understood. Some proRussia interpretation is evident, but it gives the objective reader on the USSR, understanding and a comparison to USA technology.

Please send me _..... copies of FROM SCIENTIFIC SEARCH

TO ATOMIC INDUSTRY

@ 17.90 each .... TOTAL COST \$

Payment in full enclosed

Make check payable to

AMERICAN NUCLEAR SOCIETY

$555 \mathrm{~N}$ Kensington Ave DEPT. 5

La Grange Park. Ilınois 60525

Name

Company

Address

City

State

Zip

\section{From the shadows, everyman's Maxwell}

\section{R.V. Jones}

James Clerk Maxwell: A Biography. By

Ivan Tolstoy. Pp.194. ISBN 0-86241-010-X. (Canongate, Edinburgh: 1981.) $£ 9.95$.

FOR many years there hung in one of the corridors of the Electrical Laboratory at Oxford a photographic portrait of someone whom none of the staff could identify. Presumably it had been placed there by the Professor of Physics, J.S.E. Townsend; but after his death, the identity of the portrait was forgotten. It was, in fact, of James Clerk Maxwell.

Maxwell has been almost as little known in his native Scotland, where his memory has been heavily overshadowed by that of Kelvin - and this despite the fact that Einstein himself credited Maxwell with having brought about the greatest revolution in physics since the time of Newton. Not only did Maxwell create the electromagnetic theory which led to the discovery of radio waves and of electromagnetic radiation pressure, but he also formulated his distribution law governing the molecular velocities in gases, the basis of automatic control theory and the principles of kinematic design; and he demonstrated the first colour photograph.

The current year has seen the one hundred and fiftieth anniversary of his birth on 13 June 1831, while less than two years ago there occurred the centenary of his death. The commemoration of these anniversaries has coincided with increasingly wide-spread recognition of his tremendous contributions, and with papers and books describing aspects of his life and work, notably C.W.F. Everitt's short but carefully written biography (Charles Scribner's Sons, 1975) and the exhibition of Maxwell memorabilia at last year's Royal Society conversazione.

Now we are presented with another short and very readable biography, by Ivan Tolstoy, who in his preface sets out his objective:

For physicists the name of James Clerk Maxwell ranks next to Newton and Einstein. Yet among non-scientific people Maxwell's image is surprisingly faint. It is hoped the present book will help remedy that injustice. This pretends neither to be a definitive biography, nor a work of historical scholarship. It is, rather, a book for the lay reader.

Even so, and although the author has, as he says, drawn largely on secondary sources, more specialist readers will find that he has thrown shafts of light on Maxwell's character and methods of working. And in well-drawn quotations from Maxwell's own writings he has followed one of his subject's own precepts:

It is a great advantage to the student of any subject to read the original memoirs of that subject, for science is always most completely assimilated in the nascent state.
One of the rare points in the book which could be questioned concerns the process by which Maxwell came to conceive the famous displacement current in a vacuum. Tolstoy says that "There could be no physical or logical justification for keeping the concept in the context of a vacuum". This might be true if Maxwell regarded a vacuum as containing absolutely nothing, but in his 1864 paper he stated his belief in "an aethereal medium filling space" and in its having a "small but real density"; and he evidently retained this view throughout his work, for towards the end of the Treatise of 1873 he repeated "We must therefore regard the medium as having a finite density" (para.782). So Maxwell's train of thought was both physical and logical.

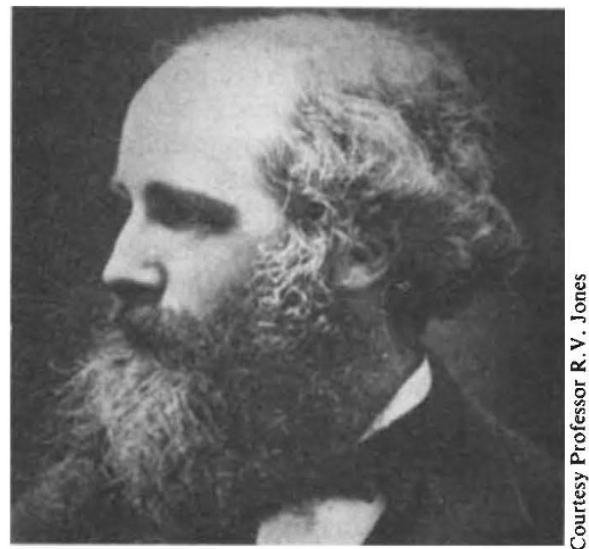

James Clerk Maxwell, the portrait that hung in the Electrical Laboratory at Oxford.

Tolstoy remarks elsewhere that "Both Einstein and Maxwell were stronger in physical intuition than in mathematics", which struck an immediate resonance in my memory with something Einstein said in conversation with F.A. Lindemann during his visit to Oxford in 1931. He was commenting on the popular description of himself as a mathematician: "I am not a mathematician. I am a physicist - if I had been a mathematician I could not have done what I have". But, of course, both Einstein and Maxwell had an impressive command of mathematics when they needed it, and Maxwell was outstandingly strong in geometrical reasoning.

Tolstoy's short account is both penetrating and sympathetic regarding Maxwell's personal relationships, of which it gives a warmly human picture; and it contains many thoughtful comments on Maxwell's physics and the context in which his advances were made. It is a book well worth reading.

R.V. Jones is Professor in the Department of Natural Philosophy at the University of Aberdeen, and author of The Complete Physicist: James Clerk Maxwell 1831-1879 (Yearbook of the Royal Society of Edinburgh, 5-23; 1980). 